\title{
Sustained-release pregabalin with methylcobalamin in neuropathic pain: an Indian real-life experience
}

\section{Yasmin U Dongre Onkar C Swami}

Unichem Laboratories Ltd, Unichem Bhavan, Mumbai, India

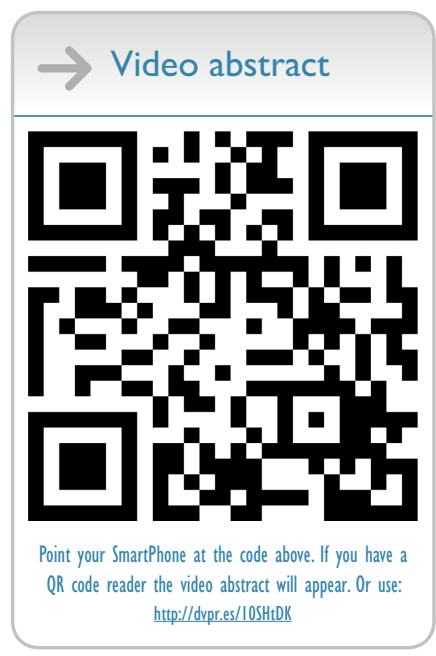

Correspondence: Onkar C Swami Medical Services, Unichem Laboratories Ltd, Unichem Bhavan, Prabhat Estate, SV Road, Jogeshwari (W), Mumbai 400102 , India

Tel +9l 2266888333

Fax +9l 2226780303

+91 2226785198

Email onkar.swami@unichemlabs.com
This article was published in the following Dove Press journal:

International Journal of General Medicine

28 May 2013

Number of times this article has been viewed

Introduction: Neuropathic pain is intense in nature and difficult to manage. Thus, the primary goal is maximum relief from pain. The aim of this study was to assess the efficacy and safety of a fixed-dose combination of sustained-release pregabalin and methylcobalamin in reducing neuropathic pain in Indian patients, in the real-life situation.

Methods: This was a multicenter, prospective, open-labeled, single-arm, observational, 14-day study. Patients received fixed dose combination of 75 or $150 \mathrm{mg}$ sustained-release pregabalin combined with $1500 \mathrm{mcg}$ immediate release methylcobalamin, depending on the clinical requirement. Data was collected for pain reduction and other positive and negative symptoms associated with neuropathy, including hyperesthesia, paresthesia, numbness/tingling, burning sensation, muscle weakness, sleep disturbances, and impairment of movement. Pain intensity was measured on a ten-point visual analog scale (VAS) (0 represented "no pain," and 10 represented "worst pain ever"). The safety of the drug was also evaluated throughout the study duration. Data was analyzed using appropriate statistical methods.

Results: The overall reduction in mean VAS score over 14 days was $72.3 \%$. The reduction in mean VAS score was significant as early as the first week. Both positive and negative symptoms of peripheral neuropathy were significantly improved in $>50 \%$ patients within the 2 weeks. Giddiness (4.7\%), followed by sedation (3.6\%), dizziness (2.9\%), drowsiness (2.3\%), and nausea $(2.3 \%)$ were the most commonly observed adverse effects. The overall efficacy and tolerability was rated as good to excellent by $>95 \%$ of the investigators and patients.

Conclusion: Fixed dose combination of sustained-release pregabalin and methylcobalamin significantly reduced neuropathic pain, with significant improvement in both the positive and negative symptoms associated with neuropathy, in Indian patients and was well tolerated.

Keywords: pregabalin, sustained release, methylcobalamin, peripheral neuropathy, India

\section{Introduction}

The International Association for the Study of Pain defines neuropathic pain (NP) as pain "initiated or caused by a primary lesion or dysfunction in the nervous system." The characteristic abnormality in NP is a lesion or dysfunction of the sensory pathway, with associated hyperexcitability of the denervated zone. ${ }^{2}$

The pain can be of high intensity and long duration and can have a profound effect on the mood, personality, and social relationships of a person. Long duration of pain causes concomitant depression, fatigue, anxiety, sleep disturbances, and decreased overall physical functioning. ${ }^{3}$ Peripheral neuropathy has various etiologies, which also differ depending on the location of the nerve damage. A single mechanism can rarely be claimed for the development of signs and symptoms of NP. ${ }^{4}$ 
Peripheral neuropathy is very common in type 2 diabetes mellitus (T2DM), with diabetic peripheral neuropathy (DPN) affecting up to half of the patients suffering from diabetes. One study, conducted in the rural part of India, reported peripheral neuropathy (PN) as the most common complication of diabetes mellitus, affecting around $60 \%$ of patients. ${ }^{5}$ Apart from pain, another important clinical manifestation of DPN is insensitivity, which increases the risk of burns, injuries, and foot ulcerations. Poor glycemic control, duration of diabetes, hyperlipidemia (particularly hypertriglyceridemia), elevated albumin excretion rate, and obesity have been implicated as risk factors for the development of DPN. ${ }^{6}$

The management of NP is difficult due to the inadequacies of the existing therapeutic options. Even the well-established drugs used in the management of peripheral neuropathy have limitations with respect to dosing regimen, unpredictable effectiveness, delay in the onset of the analgesic effects, and tolerability.

Pregabalin, a member of the gamma aminobutyric acid class, is considered as one of the first-line drugs for the treatment of NP. It has a high affinity for the auxiliary $\alpha 2 \delta$ subunits of the voltage-gated calcium channel and thus blocks $\mathrm{Ca}^{2+}$ influx into nerve terminals, which leads to reduced transmitter release. Reported evidence suggests that pregabalin is effective for both central and peripheral NP and that it achieves rapid pain reduction. Pain reduction can be achieved as early as the first week after initiating the treatment, with improvement that is dose related from 150 to $600 \mathrm{mg} /$ day. Pregabalin has also been shown to have beneficial effects on sleep and mood disturbances. ${ }^{3}$

Methylcobalamin is an essential element in the synthesis of the myelin sheath and in the maintenance of nerve function. The myelin sheath provides insulation to the nerve and aids in the proper and rapid conduction of impulses along the nerve. ${ }^{7,8}$ A deficiency of vitamin $B_{12}$ results in damage to the myelin sheath. Methylcobalamin has an important role in the regeneration of myelin sheath and helps to restore the function of the nerve in neuropathy., 10

There is limited published data on the fixed dose combination (FDC) of pregabalin and methylcobalamin in immediate release or as a sustained release formulation. The objective of the present study was to assess the efficacy and safety of the FDC of sustained-release pregabalin and immediate release methylcobalamin (PGM-SR) in reducing $\mathrm{NP}$ in a real-life situation, in Indian patients.

\section{Materials and methods}

PRECISE II (PREgabalin sustained release Combination In Several Endpoints) was a multicenter, prospective, open-labeled, single-arm, observational, 14-day study conducted in routine clinical practice. Adult patients with PN who were clinically suitable for the prescription of PGM-SR were enrolled and received Pregaba-M SR 75/150 FDC of sustained-release pregabalin $75 \mathrm{mg}$ or $150 \mathrm{mg}$ combined with methylcobalamin $1500 \mathrm{mcg}$ immediate release (Unichem Laboratories Ltd, Mumbai, India) therapy for 14 days.

Patients with known contraindications, based on the prescribing information of the product, were excluded from the study.

The study was conducted in compliance with the principles of the Declaration of Helsinki, the International Conference on Harmonisation of Technical Requirements for Registration of Pharmaceuticals for Human Use, and local regulatory guidelines, ie, Schedule Y. ${ }^{11,12}$ This was a noninterventional study of a pharmaceutical product already approved by the local regulatory authority and available by prescription. Therefore, verbal informed consent was obtained from each patient before their participation in the study.

The patients received Pregaba-M SR 75 once daily (OD) or twice daily (BD), or received Pregaba-M SR $150 \mathrm{OD}$, depending on the clinical requirement. Investigators were allowed to prescribe other necessary medications for comorbid conditions, provided these did not interfere with the study medication. There was a provision for rescue medication for pain, if required.

Demographic data was collected at the baseline visit. Pain was analyzed on a ten-point visual analog scale (VAS) (where 0 was ranked as "no pain," and 10 was ranked as "worst pain ever") and was the primary efficacy parameter. The proportion of patients having hyperesthesia, paresthesia, numbness/tingling, burning sensation, muscle weakness, sleep disturbance, and impairment of movement/ambulation were recorded. Both the primary and secondary efficacy parameters, dose of PGM-SR, and concomitant medications used were measured at the three study visits, on day 0 , day 7 , and day 14 .

After 14 days of therapy, based on the clinical condition, subjects were either continued on PGM-SR therapy or the therapy was withdrawn, at the discretion of the investigator.

Safety and tolerability were assessed continuously during the study period. Adverse events were reported to the investigators during each of the visits or any time during the course of the therapy.

\section{Statistical analysis}

The present study was analyzed using descriptive statistical analysis. Continuous data results were presented as mean with standard deviation, and categorical data results were described in frequency and percentage. The Chi-square test 
was estimated for changes in the proportion of cases with positive and negative symptoms, and analysis of variance with Kruskal-Wallis test were applied to assess the changes in pain score from baseline to the end of treatment. The significance level was set at $P<0.05$ for all the analyses.

\section{Sample size}

In this study, more than 300 evaluable patients were adequate to provide $90 \%$ power to assess a mean change of 2.25 with standard deviation of 2.0, from baseline to the end of 14 days, at a $5 \%$ level of significance.

\section{Results}

A total of 384 patients were enrolled and received the PGM-SR for 14 days. The demographic data at baseline is presented in Table 1.

The treatment with PGM-SR reported significant improvement in pain within 1 week $(P<0.05)$ and which continued further over day $14(P<0.05)$, compared with baseline (Table 2). Within a short span of 2 weeks, a $72.3 \%$ reduction in the VAS score for pain was noted.

There was significant reduction $(P<0.05)$ in the proportion of patients having negative and positive symptoms associated with neuropathy, like hyperesthesia, paresthesia, tingling/numbness, burning sensation, muscle weakness, sleep disturbance, and movement/ambulation impairment, over the 14 days of therapy, as compared with baseline (Table 3). Within 14 days, all the symptoms except for muscle weakness showed improvement, in $>50 \%$ patients. No significant improvement in cranial muscle involvement and tendon reflexes were reported.

As per the global assessment of efficacy, $99 \%$ of investigators and $97 \%$ of patients rated PGM-SR as good to excellent, and similarly, $99 \%$ of investigators and patients reported good to excellent tolerability.

The most common adverse effects (AE) occurring with a frequency of $>2 \%$ were giddiness $(4.7 \%)$, followed by

Table I Demographic profile

\begin{tabular}{ll}
\hline Parameter & Observed value \\
\hline No of patients & 384 \\
Age (yrs), (mean \pm SD) & $48.1 \mathrm{I} \pm 12.4 \mathrm{I}$ \\
Sex N (\%), male:female & $62.3: 37.7$ \\
Duration of disease (years), (mean $\pm \mathrm{SD})$ & $1.8 \mathrm{I} \pm 3.8$ \\
Patients with history of smoking, \% (n/N) & $32.5(1 \mathrm{I} / 345)$ \\
Patients with history of alcohol, \% (n/N) & $21.8(72 / 33 \mathrm{I})$ \\
Patients with T2DM, \% (n/N) & $15.9(6 \mathrm{I} / 384)$ \\
Patients with essential hypertension, \% $(\mathrm{n} / \mathrm{N})$ & $12.8(49 / 384)$ \\
\hline
\end{tabular}

Notes: $n=$ number of subjects; $N=$ total number of subjects evaluated. Abbreviations: SD, standard deviation; T2DM, type 2 diabetes mellitus.
Table 2 Improvement in pain score over the treatment, compared with baseline

\begin{tabular}{llll}
\hline Evaluation day & $\mathbf{N}$ & $\begin{array}{l}\text { Pain Score on VAS } \\
\text { (mean } \pm \text { SD) }\end{array}$ & \% change \\
\hline Baseline & 359 & $06.32 \pm 02.55$ & - \\
7 & 333 & $03.82 \pm 02.25^{*}$ & 39.6 \\
14 & 301 & $01.75 \pm 01.90^{*}$ & 72.3 \\
\hline
\end{tabular}

Notes: By analysis of variance (ANOVA) Kruskal-Wallis test, ${ }^{*} P<0.05$.

Abbreviations: SD, standard deviation; VAS, visual analog scale; N, total number of subjects evaluated.

sedation (3.6\%), dizziness (2.9\%), drowsiness (2.3\%), and nausea $(2.3 \%)$.

Other adverse events reported with an incidence of $<2 \%$ were vertigo, vomiting, dry mouth, constipation, and edema. All AE were mild to moderate in severity, except for giddiness, which was reported as severe in one patient. PGM-SR $75 \mathrm{mg} /$ day was the most frequently prescribed dose.

\section{Discussion}

PN pain is characterized by positive and negative symptoms. Positive symptoms are caused by the hyperexcitability of the nerves and include pain, paresthesia, dysesthesia, and spasm. Negative symptoms are the result of reduced impulse conduction along the nerve and include hypoesthesia, anesthesia, and weakness. ${ }^{13}$

Among the various causes for PN, T2DM is the most common cause, with an incidence as high as one in every four patients with T2DM. ${ }^{14,15}$

NP severely affects the quality of life and is often challenging for clinical management. The current treatment options for NP include tricyclic antidepressants, serotonin/norepinephrine reuptake inhibitors, topical (5\%) lidocaine patch, antiepileptic drugs (like gabapentin, pregabalin, carbamazepine, topiramate, and lamotrigine), topical capsaicin, and opioids. ${ }^{15}$ The American Academy of Neurology, the American Association of Neuromuscular and Electrodiagnostic Medicine, and the American Academy of Physical Medicine and Rehabilitation, in their joint evidence-based guideline (2010), reported that pregabalin was established to be effective and recommended that it be offered for relief of painful diabetic neuropathy (Level A recommendation). ${ }^{16}$

However, pregabalin has the limitation of having a short half-life (5-6.5 hours), which necessitates frequent administration of the drug and this might reduce patient compliance, especially when used for long term therapy. In this scenario, usage of pregabalin in a sustained-release formulation may be a good option to overcome abovementioned limitation.

Metformin, a first-line drug in T2DM, causes vitamin $\mathrm{B}_{12}$ deficiency in chronic use and thus can cause or aggravate neuropathy in patients with T2DM. ${ }^{17}$ As methylcobalamin 
Table 3 Improvement in positive and negative symptoms associated with PN, compared with baseline

\begin{tabular}{lllll}
\hline Parameter & Baseline patients (\%) & At day 7 patients (\%) & At day I4 patients (\%) & P-value \\
\hline Hyperesthesia & 48.2 & $36.9^{*}$ & $22.4^{*}$ & $<0.05$ \\
Paresthesia & 55.1 & $39.0^{*}$ & 26. F $^{*}$ & $<0.05$ \\
Numbness/tingling & 81.0 & $57.6^{*}$ & $34.8^{*}$ & $<0.05$ \\
Burning sensation & 63.2 & $45.7^{*}$ & $24.8^{*}$ & $<0.05$ \\
Muscle weakness & 27.3 & 22.3 & $16.8^{*}$ & $<0.05$ \\
Sleep disturbance & 55.6 & $27.1^{*}$ & $15.7^{*}$ & $<0.05$ \\
Movement/ambulation impairment & 41.7 & $25.4^{*}$ & $15.4^{*}$ & $<0.05$ \\
\hline
\end{tabular}

Notes: By Chi-Square test; *P-value $<0.05$; "compared with baseline.

Abbreviation: PN, peripheral neuropathy.

plays an important role in the regeneration of the myelin sheath, the addition of the same to pregabalin may restore the function of nerves, in addition to relieving the pain.

However, there is a scarcity of clinical data on the efficacy and safety of these FDCs of pregabalin and methylcobalamin. The previously published PRECISE (PREgabalin Combination In Several Endpoints) study reported that a FDC of pregabalin immediate release and methylcobalamin was effective and well tolerated in Indian patients with various neuropathies and orthopedic conditions. ${ }^{18}$ In a recently published report, Prabhoo et $\mathrm{l}^{19}$ reported the efficacy and tolerability of the FDC of pregabalin and methylcobalamin in NP. ${ }^{19}$

To the best of our knowledge, PRECISE-II is the first clinical report on the FDC PGM-SR.

There was a significant reduction in the VAS score as early as the first week of treatment. The relief from pain continued to be significant over the 2-week period, changing from severe at baseline to mild at 14 days. ${ }^{20}$

Patients treated with PGM-SR demonstrated improvement in both positive as well as negative symptoms of peripheral neuropathy. The improvements were consistent irrespective of the cause of neuropathy including DPN, postherpetic neuralgia, and other musculoskeletal PN conditions.

PGM-SR was generally well tolerated. Safety results were in accordance with the known safety profile of the components of PGM-SR.

The most commonly prescribed dosage of PGM-SR in the PRECISE II study was pregabalin $75 \mathrm{mg}$ /day, contrary to the recommended dose range of pregabalin (150-600 mg/day). This may have been due to the fact that the optimal dose titration was not achieved during the short duration of the study, that there was over-enthusiastic dispensation of rescue medication, or that the lower dose of pregabalin may be adequate for treatment of NP, for Indian patients. Nonetheless, the efficacy of such a low dose pregabalin as was observed in this study should be further evaluated in a well-designed multicenter, randomized, controlled clinical trial.
The lower incidence of adverse effects, such as sedation ( $38.50 \%$ vs $3.6 \%$ ) and giddiness (36.73\% vs $4.7 \%$ ), in the PRECISE II compared with the PRECISE study can be attributed to the sustained release formulation and the use of a lower dose of pregabalin in majority of patients in the present study. ${ }^{17}$

The level of satisfaction with PGM-SR expressed by the patients as well as the investigators can be attributed to the low incidence of adverse events and the convenience of once daily dosing that was possible with the sustainedrelease formulation used. This may be useful for increasing the compliance in chronic conditions, like DPN.

The results of this study must be interpreted cautiously considering the many important limitations: an open-label study, observational in nature; small sample size; lack of details of the concomitant medications; and the prescription of the PGM-SR formulation for off label indications, like musculoskeletal PN conditions. We accept that our study design was not as robust as contemporary studies in pain management. Additionally there was missing data for some of variables at some of the visits.

The effectiveness of the lower dose of pregabalin in reducing both the positive and negative symptoms of neuropathy in Indian patients, and the lower incidence of adverse events observed with the sustained-release formulation reported in this study must be established in large, multicenter, randomized, controlled study.

\section{Conclusion}

The FDC of PGM-SR significantly reduced NP at a lower than recommended dose, with significant improvement in both the positive and negative symptoms associated with neuropathy, in Indian patients. This FDC was well tolerated in Indian patients.

\section{Acknowledgments}

This study was sponsored by Unichem Laboratories Limited, Mumbai, India. We gratefully acknowledge and thank the PRECISE II study Investigators group: Dr Piyush Shah, Ahmedabad; Dr Parth N Patel, Ahmedabad; Dr SV Dubal, Bhavnagar; 
Dr Sanjay Parikh, Bhavnagar; Dr Kalpesh Zala, Jamnagar; Dr Dhaivat Nanvati, Baroda; Dr Jay Vankawala, Surat; Dr Apurva Kapadia, Surat; Dr Kiran Jadhav, Valsad; Dr Vinit Minocha, Panipat; Dr Sanjay Kr Pal, Pondicherry; Dr SM Ilanchezhian, Nagercoil; Dr Rishi Raj Shokeen, Delhi; Dr K Khastgir, Delhi; Dr Ashish Rohtagi, Delhi; Dr Monika Basudev, Delhi; Dr Rajeev Nigam, Delhi; Dr Lokesh Bansal, Delhi; Dr Munindra A Raval, Hyderabad; Dr D Rajaiah, Kurnool; Dr AM Ilias Basha, Kurnool; Dr SM Abid, Cuddapah; Dr S Krishna Reddy, Nellore; Dr RN Jaiswal, Indore; Dr OP Gupta, Ratlam; Dr Vivek Jain, Ujjain; Dr RK Nawlakhe, Khandwa; Dr DK Jain, Gwalior; Dr Rahul Deo, Sagar; Dr PK Verma, Rewa; Dr Gaurav Rohatgi, Kota; Dr GP Kejriwal, Kolkata; Dr Tanmay Das, Kolkata; Dr Uttam Kumar Chaubey, Kolkata; Dr Chhabi Biswas, Kolkata; Dr Malay Sarkar, N24paragan; Dr Kaushik Chandwani, N24paragan; Dr Arindam Chattopadhyay, Hooghly; Dr DK Ghosh, Hooghly; Dr S. Mukherjee, Kolkata; Dr Arbind Shah; Kolkata; Dr Anup Agrawal, Lucknow; Dr Pradeep Kumar, Mirzapur; Dr Sanjeev Gupta, Mumbai; Dr Sameer Sheikh, Mumbai; Dr Mihir Patel, Mumbai; Dr Pradyot Behera, Cuttack; Dr Ramniwas Jain, Bhubaneshwar; Dr NK Verma, Dhanbad; Dr Achinto Mandal, Dhanbad; Dr Mani Bhushan, Chapra; Dr Vivek Mahajan, Nagpur; Dr R Waman Rao Khapre, Gondia. We also thank Mr Kailas Gandewar for his help in statistical analysis.

\section{Disclosure}

Dr Dongre and Dr Swami are full-time employees of Unichem Laboratories Limited, which sponsored this study. The authors report no other conflicts of interest.

\section{References}

1. Merskey H, Bogduk N. Classification of Chronic Pain: Descriptions of Chronic Pain Syndromes and Definitions of Pain Terms, 2nd ed. Seattle, WA: IASP Press; 1994.

2. Jensen TS, Gottrup H, Sindrup SH, Bach FW. The clinical picture of neuropathic pain. Eur J Pharmacol. 2001;429(1-3):1-11.

3. Anastassiou E, Iatrou CA, Vlaikidis N, et al; ATLAS Investigators. Impact of pregabalin treatment on pain, pain-related sleep interference and general well-being in patients with neuropathic pain: a non-interventional, multicentre, post-marketing study. Clin Drug Investig. 2011;31(6):417-426.

4. Jensen TS, Baron R. Translation of symptoms and signs into mechanisms in neuropathic pain. Pain. 2003;102(1-2):1-8.
5. Vaz NC, Ferreira A, Kulkarni M, Vaz FS, Pinto N. Prevalence of diabetic complications in rural Goa, India. Indian J Community Med. 2011;36(4):283-286.

6. Tesfaye S, Selvarajah D. Advances in the epidemiology, pathogenesis and management of diabetic peripheral neuropathy. Diabetes Metab Res Rev. 2012;28 Suppl 1:S8-S14.

7. Minn YK, Kim SM, Kim SH, Kwon KH, Sunwoo IN. Sequential involvement of the nervous system in subacute combined degeneration. Yonsei Med J. 2012;53(2):276-278.

8. Stabler SP. Clinical practice. Vitamin $\mathrm{B}_{12}$ deficiency. $N$ Engl J Med. 2013;368(2):149-160.

9. Okada K, Tanaka H, Temporin K, et al. Methylcobalamin increases Erk1/2 and Akt activities through the methylation cycle and promotes nerve regeneration in a rat sciatic nerve injury model. Exp Neurol. 2010;222(2):191-203.

10. Yamatsu K, Yamanishi Y, Kaneko T, Ohkawa I. Pharmacological studies on degeneration and regeneration of the peripheral nerves. (2) Effects of methylcobalamin on mitosis of Schwann cells and incorporation of labeled amino acid into protein fractions of crushed sciatic nerve in rats. Nihon Yakurigaku Zasshi. 1976;72(2):269-278. Japanese.

11. International Conference on Harmonisation. ICH Harmonised Tripartite Guideline. Available at http://www.ich.org/fileadmin/Public_Web_Site/ ICH_Products/Guidelines/Efficacy/E6_R1/Step4/E6_R1_Guideline. pdf. Accessed April 25, 2013.

12. Schedule Y. Requirements and guidelines for permission to import and/ or manufacture of new drugs for sale or to undertake clinical trials. Available at http://cdsco.nic.in/html/schedule-y\%20(amended\%20 version-2005)\%20original.htm. Accessed April 25, 2013.

13. Nee RJ, Butler D. Management of peripheral neuropathic pain: Integrating neurobiology, neurodynamics, and clinical evidence. Physical Therapy in Sport. 2006;7(1):36-49.

14. Head KA. Peripheral neuropathy: pathogenic mechanisms and alternative therapies. Altern Med Rev. 2006;11(4):294-329.

15. Smith HS, ArgoffCE. Pharmacological treatment of diabetic neuropathic pain. Drugs. 2011;71(5):557-589.

16. Bril V, England J, Franklin GM, et al; American Academy of Neurology; American Association of Neuromuscular and Electrodiagnostic Medicine; American Academy of Physical Medicine and rehabilitation. Evidence-based guideline: Treatment of painful diabetic neuropathy: report of the American Academy of Neurology, the American Association of Neuromuscular and Electrodiagnostic Medicine, and the American Academy of Physical Medicine and Rehabilitation. Neurology. 2011;76(20):1758-1765.

17. Mahajan R, Gupta K. Revisiting metformin: Annual vitamin B12 supplementation may become mandatory with long-term metformin use. J Young Pharm. 2010;2(4):428-429.

18. Joshi KK, Mukaddam Q. PRECISE Survey-PREgabalin Combination In Several Endpoints survey. IMG. 2010:302-309.

19. Prabhoo R, Panghate A, Dewda RP, More B, Prabhoo T, Rana R. Efficacy and tolerability of a fixed dose combination of methylcobalamin and pregabalin in the management of painful neuropathy. NAm J Med Sci. 2012;4(11):605-607.

20. Kastler A, Aubry S, Piccand V, Hadjidekov G, Tiberghien F, Kastler B. Radiofrequency neurolysis versus local nerve infiltration in 42 patients with refractory chronic inguinal neuralgia. Pain Physician. 2012;15(3):237-244.

\section{Publish your work in this journal}

The International Journal of General Medicine is an international, peer-reviewed open-access journal that focuses on general and internal medicine, pathogenesis, epidemiology, diagnosis, monitoring and treatment protocols. The journal is characterized by the rapid reporting of reviews, original research and clinical studies across all disease areas.

\section{Dovepress}

A key focus is the elucidation of disease processes and management protocols resulting in improved outcomes for the patient.The manuscript management system is completely online and includes a very quick and fair peer-review system. Visit http://www.dovepress.com/ testimonials.php to read real quotes from published authors. 